BNL- 76988-2006-CP

\title{
Electron Beam Generation and Transport for the RHIC Electron Cooler
}

\author{
J. Kewisch, I. Ben-Zvi, X.Y. Chang, \\ Particle Accelerator Conference, pp. 2774-2776 \\ Knoxville, TN \\ May 16-20, 2005
}

August 2006

Collider-Accelerator Department
Brookhaven National Laboratory
P.O. Box 5000
Upton, NY 11973-5000
www.bnl.gov

\begin{abstract}
Notice: This manuscript has been authored by employees of Brookhaven Science Associates, LLC under Contract No. DE-AC02-98CH10886 with the U.S. Department of Energy. The publisher by accepting the manuscript for publication acknowledges that the United States Government retains a non-exclusive, paid-up, irrevocable, world-wide license to publish or reproduce the published form of this manuscript, or allow others to do so, for United States Government purposes.
\end{abstract}




\section{DISCLAIMER}

This report was prepared as an account of work sponsored by an agency of the United States Government. Neither the United States Government nor any agency thereof, nor any of their employees, nor any of their contractors, subcontractors, or their employees, makes any warranty, express or implied, or assumes any legal liability or responsibility for the accuracy, completeness, or any third party's use or the results of such use of any information, apparatus, product, or process disclosed, or represents that its use would not infringe privately owned rights. Reference herein to any specific commercial product, process, or service by trade name, trademark, manufacturer, or otherwise, does not necessarily constitute or imply its endorsement, recommendation, or favoring by the United States Government or any agency thereof or its contractors or subcontractors. The views and opinions of authors expressed herein do not necessarily state or reflect those of the United States Government or any agency thereof. 


\title{
ELECTRON BEAM GENERATION AND TRANSPORT FOR THE RHIC ELECTRON COOLER*
}

\author{
Jörg Kewisch, Ilan Ben-Zvi, Xiangyun Chang \\ BNL, Upton, NY, USA
}

\section{Abstract}

An electron cooler, based on an Energy Recovery Linac (ERL) is under development for the Relativistic Heavy Ion Collider (RHIC) at Brookhaven National Laboratory. This will be the first electron cooler operating at high energy with bunched beams. A better understanding of the cooling process and more accurate measurements of Intra Beam Scattering in RHIC have imposed increased requirements on the electron accelerator: Besides a doubling of the bunch charge to 20 $\mathrm{nC}$, the strength of the cooling solenoid was increased five-fold to 5 Tesla. The magnetic field on the cathode should be increased to 500 Gauss to match the magnetization required in the cooling solenoid. This paper reports the measures taken to minimize the electron beam emittance in the cooling section.

\section{INTRODUCTION}

Electron cooling is the main element of the luminosity upgrade of RHIC. The cooler is based on an energy recovery linac as shown in Fig. 1. A train of short electron bunches is created in a photo-cathode RF gun and accelerated in a superconductive linac to an energy of 55 $\mathrm{MeV}$. The logitudinal phase space using an arc and a debunching cavity. They are then merged with the ions in RHIC where a 5 Tesla solenoid filed is aiding the cooling process. After separation from the ions the electron bunch length is compessed in a second arc to its original length and decelerated in the linac. The kinetic energy is put back into the cavity and is used to accelerate the next electron bunch. After decelleration the electrons are dumped.

Each bunch travels through the system only once. The emmitance of the electron beam can therefore be much smaller than the equilibrium emmittance of an equivalent storage ring and problems with resonances are minimized.

Since the thermal emittance at the cathode is small the emittance of the electron beam in the cooler section is determined by space charge effects and nonlinearities of the beam transport elements. It is therefore important to compensate these effects as well as possible.

As a strong solenoid field is used in the cooling section the electron beam must be "magnetized" for a minimum temperature inside the cooler. The fringe field of the solenoid produces a large angular momentum. The beam must therefore enter the solenoid with the opposite angular momentum.

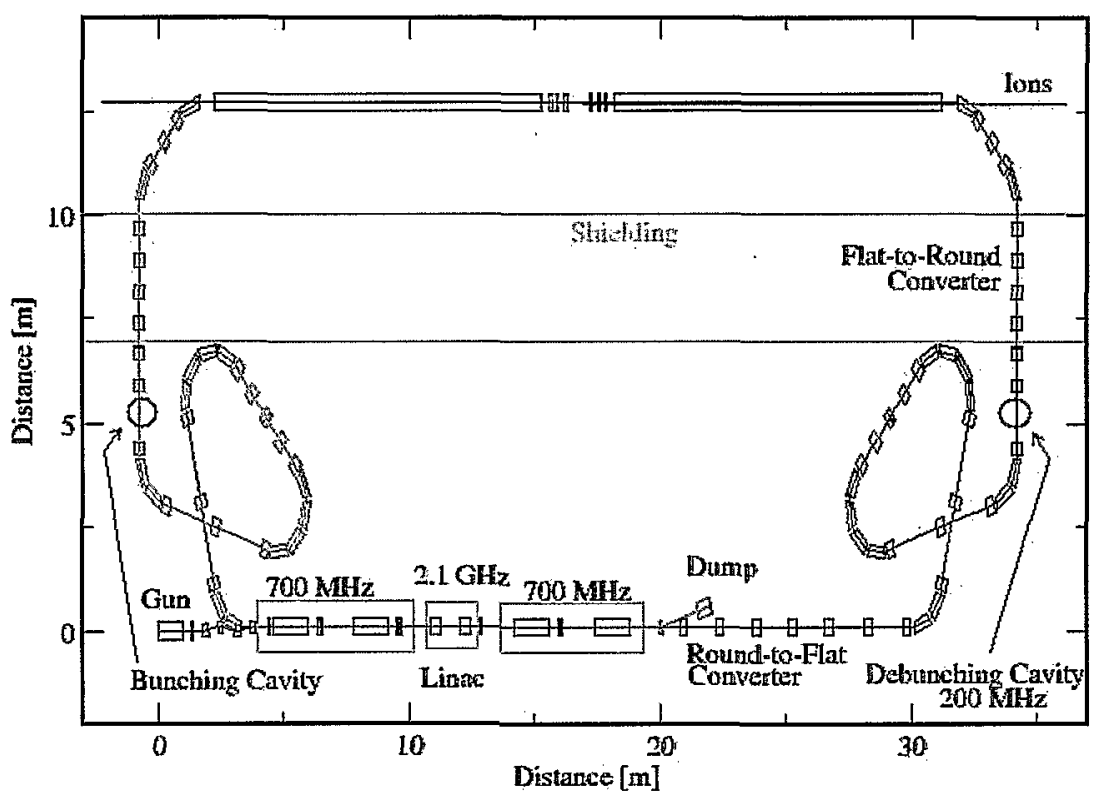

Figure 1: Layout of the RHIC cooler

\footnotetext{
" Work performed under the auspices of the U.S. Department of

Energy.
} 
According to Busch's Theorem the only way to create such a beam is to immerse the cathode in an equivalent longitudinal field:

$$
r^{2} \cdot \Theta^{\prime}+r^{2} \cdot \frac{e}{p} \cdot B=r_{0}^{2} \cdot \frac{e}{p} \cdot B_{0}
$$

where $r$ is the beam radius, $B$ is the longitudinal field, $\Theta^{\prime}$ is the rotation speed and the subscript 0 indicates the value on the cathode.

Although the emittance of the beam in a frame rotating with $\Theta^{\prime}$ is small, the emittance in the lab frame is large (1500 $\mathrm{mm} \mathrm{mrad})$. Small changes of the emittance in the lab frame result in large changes in the rotating frame and the cooling section.

On the way from the gun to the cooling solenoid three things have to be accomplished:

- The low energy beam coming from the gun is merged with the high energy beam returning from the cooler for energy recovery.

- The beam must be accelerated.

- The bunch must be stretched to a length of at least $12.5 \mathrm{~cm}$ rms, so that the Debye shielding does not limit the friction force. Through bunch rotation the momentum spread is also reduced.

We investigated the emittance degrading effects and their compensation. We will first consider the stretcher and establish a correction scheme. Then we discuss who this correction can be applied to the low energy part of the cooler.

\section{OPTIMIZATION OF THE STRETCHER}

At the end of the linac the beam has an energy of 55 $\mathrm{MeV}$ with a spread of $4 \cdot 10^{-4}$ and arms bunch length of 6.5 $\mathrm{mm}$. The thermal emittance is $38 \mathrm{~mm}$ mrad. A loop with a total deflection of 450 degrees provides a large R56 matrix element to stretch the beam. By miss-phasing the last linac cavity the energy spread is increased to $7.2 \cdot 10^{-4}$, so that the required bunch length is obtained. The bunch is then rotated using a $200 \mathrm{MHz}$ cavity, so that the final energy spread is $1 \cdot 10^{-4}$. There are three sources of nonlinearities:

Chromaticity: It is not sufficient to make the phase advance independent of the momentum. The requirement is that the magnetization is not disturbed:

$$
\frac{d\left(x^{\prime}-\Theta^{\prime} \cdot y\right)}{d \delta}=0 \text { and } \frac{d\left(y^{\prime}+\Theta^{\prime} \cdot x\right)}{d \delta}=0
$$

where $\delta=\frac{\Delta \dot{p}}{p}$. In order to correct the momentum dependence eight families of sextupoles would be necessary, introducing strong transverse non-linearities. It is therefore better to use a small momentum spread and a large R56 matrix element in the spreader, so that the natural chromaticity is small and does not need to be corrected.

Multipoles: Even without chromaticity correction a multipole correction is necessary. The strongest contribution comes from the dipoles, where geometric effects not only cause the weak focusing but also a sextupole component. The best way to deal with this effect is a local correction. By introducing a sextupole in every dipole the emittance growth is minimized.

Space Charge: Space charge is even at $55 \mathrm{MeV}$ a contributor to the emittance degradation. The most important effect is the coupling of the longitudinal and transversal motion through space charge. As the electrons travel trough the high dispersion region of the spreader the space charge is increasing the momentum of the electrons in the head of the bunch and decelerating the tail. Although the dispersion of the stretcher is matched this effect causes a change of the betatron horizontal motion and destroys the magnetization.

We have investigated two different methods to remedy the emittance degradation. The first method converts the magnetized beam into a flat beam before the spreader and converts it back afterwards. This method was described by Brinkmann et al. [1]. The vertical emittance after the conversion is proportional to the thermal emittance of the magnetized beam; the horizontal emittance will be twice the real emittance of the magnetized beam. As the longitudinal space charge changes only the horizontal motion, the growth of the thermal emittance is minimized.

The second method, described by Kayran and Litvinenko [4,5], decouples longitudinal and transverse motion in the presence of space charge. We used an approximate expression to describe the effect of the space charge on the electron momentum:

$$
\frac{d \delta}{d s}=\frac{f\left(\xi_{0}\right)}{\sigma_{l}(s)}
$$

where $\sigma_{l}(s)$ is the electron bunch length and $\xi_{0}$ is initial longitudinal position of the electron in the bunch. The method defines four conditions in the form of integrals, which should be made equal zero $[4,5]$. Two of these conditions are equivalent to a standard achromatic condition.

The two additional conditions can be satisfied by using two loops as shown in figure 2. The ratio of the dispersion amplitude in the loops (shown in red and blue) and the phase advance between the loops (shown in green) provide the variables to make the integrals zero.



Figure 2: Double loop layout 
The computer code PARMELA [6] was used to simulate the different designs. The result is shown in table 1.

Table 1: Emittance in the cooling section with different compensation methods.

\begin{tabular}{|l|l|c|}
\hline Stretcher & Beam & Cooler Emittance \\
\hline Single Loop & Round & $118 \mathrm{~mm} \mathrm{mrad}$ \\
\hline Single Loop & Flat & $83 \mathrm{~mm}$ mrad \\
\hline Double Loop & Round & $70 \mathrm{~mm}$ mrad \\
\hline Double Loop & Flat & $75 \mathrm{~mm}$ mrad \\
\hline
\end{tabular}

It shows that the compensation with a double loop is more effective than the flat beam and that it does not help to do both. A hand-waving argument is that the flat beam has a larger density, making transverse space charge effects more important.

\section{EMITTANCE COMPENSATION AND MERGING SYSTEM}

Emittance compesation was described by Serafini and Rosenzweig [2] but needs to be amended. Because of the magnetization of the beam it is not only necessary to align the phase advance of the longitudinal slices of the beam, but also to minimize the variation of the beam radius with the length of the bunch. Otherwise one can only compesate the avarage angular momentum of the beam, leaving different logitudinal slices with significant angular momentum.

It turns out that the the variation of the radius can not be compensated at the same time as the phase [3]. The best one can do is make the beam radius large, so that in enveloppe equation the space charge force dominates over the angular momention term:

$$
\frac{K_{s}}{\beta^{3} \gamma^{3} \sigma} \approx\left(\frac{\mathrm{p}_{\theta}}{\beta \gamma m c}\right)^{2} \frac{1}{\sigma^{3}}
$$

where $K_{s}=I / 2 I_{0}$ and $\mathrm{I} 0$ is $17000 \mathrm{~A}$.

The merging system is based on dipole magnets, which deflect the low energy beam, but have little effect on the high-energy beam. Again, the effect of dispersion and space charge must be considered. Since the momentum spread is about $1 \%$ strong focusing with solenoids or quadrupoles would lead to strong chromatic effects and would impede the emittance compensation. The design used is a zigzag [4] using four dipoles instead, which fulfills eqn. 3 approximately.

\section{CONCLUSION}

Calculations of the cooling rate call for a normalized emittance of not more than $50 \mathrm{~mm}$ mrad. So far we have established in PARMELA calculations $70 \mathrm{~mm}$ mrad, based on the approximation of eqn. 3 . We are confident that with further optimizations $50 \mathrm{~mm} \mathrm{mrad}$ can be achieved.

\section{REFERENCES}

[1] R. Brinkmann et al, Phys Rev. ST Accel. Beams 4, 053501 (2001)

[2] J. Rosenzweig, L. Serafini, "Transverse particle motion in radio-frequency linear accelerators", Physical Review E, V49, 1599

[3] X. Y. Chang, "Design of electron injector system for RHIC electron cooling project and study of the secondary emission enhanced photo-cathode RF gun", Thesis, SUNY Stoneybrook, NY

[4] "Optimal merger optics and matching to the main linac", D. Kayran and V.N. Litvinenko, The 32nd Advanced ICFA Beam Dynamics Workshop on Energy Recovering Linacs, Newport News, VA, March 18-23, 2005 http://www.jlab.org/intralab/calendar/archive04/erl/w g2.html\#wg2sun

[5] Novel Method of Emittance Preservation in ERL Merging System in Presence of Strong Space Charge Forces, D. Kayran, V.N. Litvinenko, WPAP037, these proceedings

[6] L. M. Young: Parmela, LANL, LA-UR-96-1835 